\title{
Experimental investigation on the potential of the Feedback Fx-LMS Active Noise Control technology for use in industrial generator sets
}

\author{
Marco Ciampolini ${ }^{1}$, Lorenzo Bosi ${ }^{1}$, Luca Romani $^{1}$, Andrea Toniutti ${ }^{1}$, Matteo Giglioli ${ }^{2}$, \\ Paolo Maccari ${ }^{2}$, Daniele Farruggia ${ }^{2}$, and Giovanni Ferrara, ${ }^{1, *}$ \\ ${ }^{1}$ Università degli Studi di Firenze, Department of Industrial Engineering, Via di Santa Marta 3, 50134 \\ Florence, Italy \\ ${ }^{2}$ PR INDUSTRIAL s.r.l., Località Il Piano, 53031 Siena, Italy
}

\begin{abstract}
Active Noise Control (ANC) has been considered a promising technology for the abatement of acoustic noise from the mid-20th century. Feedback and Feedforward ANC algorithms, based on the destructive interference principle applied to acoustic waves, have been developed for different applications, depending on the spectrum of the noise source. Feedback ANC algorithms make use of a single control microphone to measure an error signal which is then employed by an adaptive filter to estimate the noise source and generate an opposite-phase control signal. The Fx-LMS (Filtered-X Least Mean Square) algorithm is mostly adopted to update the filter. Feedback ANC systems have proven to be effective for the abatement of low-frequency quasi-steady noises; however, different challenges must be overcome to realize an effective and durable system for high-temperature application. This paper aims at experimentally assessing the feasibility of a Feedback Fx-LMS ANC system with off-line Secondary Path estimation to be used in mid-size diesel gensets for the reduction of the exhaust noise. Several solutions are proposed, including the mechanical design, the development of the Fx-LMS algorithm in the LabVIEW FPGA programming language, and the key features required to prevent parts from thermal damage and fouling. The developed prototype was implemented on a 50-kW diesel genset and tested in a semi-anechoic chamber. The noise abatement inside the exhaust pipe and at different measurement points around the machine was evaluated and discussed, showing good potential for improving the acoustic comfort of genset users.
\end{abstract}

\section{Introduction}

The increasing demand for acoustic comfort and the more and more stringent legislation on noise pollution limits in the industrial field has moved the research toward innovative systems for noise abatement. Passive silencers, based on the combined action of noiseabsorbing material, dissipative multi-perforated sheets, and enclosed volumes built on the destructive interference of soundwaves, are the most widespread technology due to the

\footnotetext{
*Corresponding author: giovanni.ferrara@unifi.it
} 
limited costs and the wide availability of manufacturers on the market. Passive silencers however suffer for the big size which is required in order to abate low-frequency noise sources [1]. This issue is especially evident in industrial gensets, in which the most influent component of the overall noise (in terms of linear $\mathrm{dB}$ ) is the combustion-related noise [2]; in fact, the effectiveness of traditional mufflers on the low-frequency spectrum moves in an opposite way than compactness requirements.

Active Noise Control (ANC) was found to be a powerful instrument for the reduction of industrial noise pollution while limiting encumbrances of the abatement devices. ANC systems, based on the destructive interference principle, aims at attenuating an unwanted noise emitted by a general source before it reaches the area of interest. The undesired noise is reduced by emitting a sound signal with the same amplitude and opposite phase by means of an electroacoustic actuator [3]. Several ANC solutions have been tested in many industrial and automotive applications, e.g., portable generators [4], automotive [5,6] and locomotive [7] mufflers, car [8], aircraft [9] and yacht [10] cabins, ventilation ducts [11], power transformers [12], sound-proof windows [13], up to the well-known active headsets.

In ANC systems, the control signal is generated by means of an adaptive filter which records the variations of the primary noise. The finite-impulse response (FIR) filter is the simplest to be implemented [14]. Usually, the Least Mean Square algorithm is adopted to update the filter, hence the name Fx-LMS [15]. A control microphone is used to measure the residual noise; that signal is processed by the LMS algorithm to update the FIR filter.

ANC algorithms are sorted in two main classes, depending on the method of evaluation of the primary source. In Feedforward ANC algorithms (Fig. 1, left) a second (reference) microphone is used to exactly measure the noise source $x(n)$. This signal enters the adaptive filter $\mathbf{W}(z)$ generating the control signal $y(n)$. In this case the algorithm is called Broadband Feedforward [16]. A phase sensor, e.g., the Hall sensor of a flywheel, can be used instead of the reference microphone to generate a trigger signal, reducing costs, while increasing feasibility in high temperature applications (e.g., automotive exhaust). The trigger signal is used to estimate the frequency of the noise source, leading the filter to search for the control signal phase and amplitude only. This configuration is called Narrowband Feedforward [16]. In Feedback ANC algorithms (Fig. 1, right) instead the estimation of the primary noise $\hat{d}(n)$ is generated by adding the forecast of the control signal at the error microphone $\hat{y}^{\prime}(n)$ to the error signal $e(n)$ [16]. Even hybrid configurations can be developed [17].
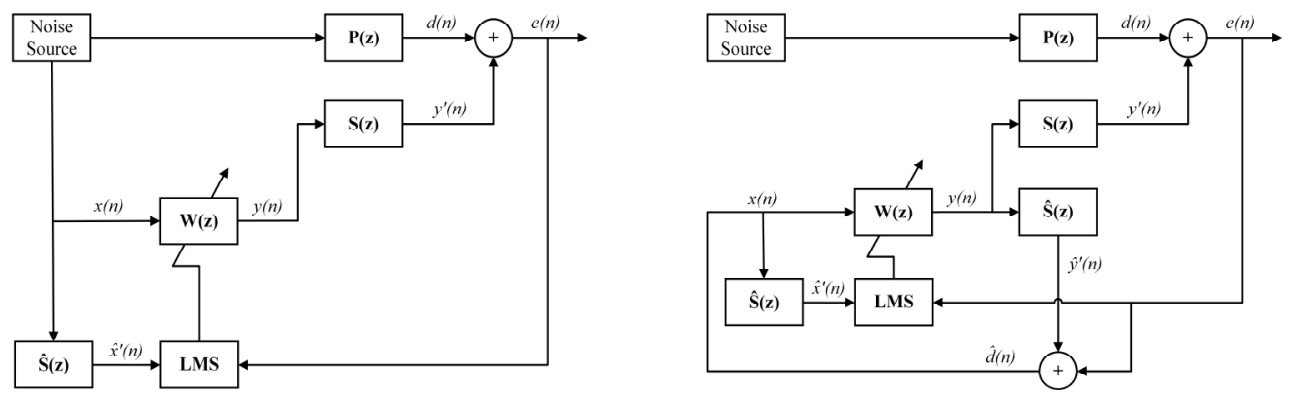

Fig. 1. Feedforward LMS ANC algorithm (left) and Feedback LMS ANC algorithm (right).

Feedback algorithms perform satisfactorily with narrowband quasi-steady noises, while Broadband Feedforward algorithms are well suited for broadband transient noises, despite the more complex layout of the system [18]. Finally, Narrowband Feedforward algorithms are the best choice for multi-tonal transient noise sources, as long as an engine speed trigger sensor signal is available [16,18]. Each method anyway requires the knowledge of the Secondary Path $\mathbf{S}(z)$, i.e., the physical and electronical delay and amplification which occur between the control signal actuator and the error microphone. In quasi-steady applications 
$\mathbf{S}(z)$ can be estimated off-line (i.e., turning off the primary noise source) by means of a dedicated algorithm [3]; on-line algorithms, in which the estimated Secondary Path $\hat{\mathbf{S}}(n)$ is real-time updated, are instead available for system plenty transient conditions $[19,20]$.

The aim of this study is to investigate the potential of adopting an ANC system on industrial mid-size gensets in order to reduce combustion noise and its harmonics. Several papers investigating the use of ANC systems on engine noise are available in the Literature, showing that all the algorithms can be used in gensets (Table 1). These data will be used to evaluate the effectiveness and adequacy of the ANC system proposed by the Authors.

Table 1. Some of the experimental ANC mufflers for use in ICEs investigated in the Literature.

\begin{tabular}{|c|c|c|c|c|c|c|}
\hline Year & Ref. & $\begin{array}{c}\text { Noise } \\
\text { Source }\end{array}$ & Algorithm & $\begin{array}{c}\text { Secondary } \\
\text { Path }\end{array}$ & $\begin{array}{c}\text { Measurement } \\
\text { Station }\end{array}$ & $\begin{array}{c}\text { Overall SPL } \\
\text { Reduction }\end{array}$ \\
\hline 1991 & {$[2]$} & Genset & $\begin{array}{c}\text { Narrowband } \\
\text { Feedforward }\end{array}$ & Offline & $\begin{array}{c}\text { Ambient } 1.9 \mathrm{~m} \\
\text { from exhaust }\end{array}$ & $5.5 \mathrm{~dB}(1800 \mathrm{rpm})$ \\
\hline 1998 & {$[6]$} & $\begin{array}{c}\text { Car } \\
\text { exhaust }\end{array}$ & $\begin{array}{c}\text { Narrowband } \\
\text { Feedforward }\end{array}$ & Online & $\begin{array}{c}\text { Error mic. } \\
\text { inside duct }\end{array}$ & $5 \mathrm{~dB}(3000 \mathrm{rpm})$ \\
\hline 2000 & {$[21]$} & Engine & $\begin{array}{c}\text { Broadband } \\
\text { Feedforward }\end{array}$ & Offline & $\begin{array}{c}\text { Ambient } 0.5 \mathrm{~m} \\
\text { from exhaust }\end{array}$ & $\begin{array}{c}3.7 \mathrm{~dB}(800 \mathrm{rpm}) \\
1.9 \mathrm{~dB}(3000 \mathrm{rpm})\end{array}$ \\
\hline 2002 & {$[22]$} & $\begin{array}{c}\text { Simulated } \\
\text { car exhaust }\end{array}$ & Feedback & Offline & $\begin{array}{c}\text { Ambient } 1.5 \mathrm{~m} \\
\text { from exhaust }\end{array}$ & $6 \mathrm{~dB}$ \\
\hline 2002 & {$[7]$} & $\begin{array}{c}\text { Locomotor } \\
\text { exhaust }\end{array}$ & $\begin{array}{c}\text { Broadband } \\
\text { Feedforward }\end{array}$ & Offline & $\begin{array}{c}\text { Ambient } 2 \mathrm{~m} \\
\text { from exhaust }\end{array}$ & $\begin{array}{c}6 \mathrm{~dB}(600 \mathrm{rpm}) \\
5.5 \mathrm{~dB}(1200 \mathrm{rpm})\end{array}$ \\
\hline 2002 & {$[23]$} & $\begin{array}{c}\text { Car } \\
\text { exhaust }\end{array}$ & $\begin{array}{c}\text { Broadband } \\
\text { Feedforward }\end{array}$ & Offline & $\begin{array}{c}\text { Ambient } 1 \mathrm{~m} \\
\text { from exhaust }\end{array}$ & $\begin{array}{c}8.5 \mathrm{~dB}(2000 \mathrm{rpm}) \\
8 \mathrm{~dB}(3000 \mathrm{rpm})\end{array}$ \\
\hline 2014 & {$[24]$} & $\begin{array}{c}\text { Simulated } \\
\text { low noise }\end{array}$ & $\begin{array}{c}\text { Simplified } \\
\text { Feedback }\end{array}$ & Offline & $\begin{array}{c}\text { Error mic. } \\
\text { inside duct }\end{array}$ & $10 \mathrm{~dB}$ \\
\hline 2017 & {$[25]$} & $\begin{array}{c}\text { Simulated } \\
\text { low noise }\end{array}$ & Feedback & Offline & $\begin{array}{c}\text { Error mic. } \\
\text { inside duct }\end{array}$ & $7.9 \mathrm{~dB}$ \\
\hline 2018 & {$[26]$} & $\begin{array}{c}\text { Motorcycle } \\
\text { exhaust }\end{array}$ & Feedback & Offline & $\begin{array}{c}\text { Ambient } 1 \mathrm{~m} \\
\text { from exhaust }\end{array}$ & $\begin{array}{c}2 \mathrm{~dB}(1000 \mathrm{rpm}) \\
1.5 \mathrm{~dB}(2000 \mathrm{rpm})\end{array}$ \\
\hline
\end{tabular}

The high temperature of the exhaust gas, which may damage the reference microphone, and the requirement of a simple and cost-effective layout, push toward the use of the Feedback algorithm. This hypothesis is supported by the characteristic of engine-related genset noise in the exhaust. In fact, in accordance with [2], the exhaust sound spectrum is dominated by the noise generated at the engine firing frequency, which for a four-stroke four-cylinder inline engine running at $1500 \mathrm{rpm}$ corresponds to $50 \mathrm{~Hz}$, followed by the second harmonic $(100 \mathrm{~Hz})$ and, with a minor influence, the third harmonic $(150 \mathrm{~Hz})$. The $\mathrm{mid} /$ high frequency range of the noise spectrum is instead related to the flow turbulence, which abatement is effective by means of passive solutions [2].

This paper presents a methodology for developing and implementing a Feedback FxLMS ANC system in the exhaust pipe of mid-size generator sets. The criteria followed to implement the algorithm on a FPGA controller will be shown, together with the mechanical solutions required to prevent components from thermal and electric damage. Experimental results achieved in a semi-anechoic chamber by applying the developed ANC system on a $50-\mathrm{kW}$ four-cylinder inline diesel genset will be presented. The effectiveness and the stability of the developed solution will be discussed, while strategies for improving further noise abatement will be proposed. 


\section{Materials and methods}

\subsection{Design of the ANC FPGA code}

The ANC Fx-LMS algorithm was written in the LabVIEW FPGA language and compiled on a Xilinx Kintex-7 70T FPGA installed in a NI 9045 Compact RIO (cRIO) device. The implemented algorithm is shown in Fig. 2, followed by the constitutive equations (Eq. 1-6), in which bold symbols state for vectors. In the algorithm, the estimation of the primary source is made by adding the forecast of the control signal at the error microphone $\hat{y}^{\prime}(n)$ to the error signal $e(n)$ (Eq. 1). In accordance with [27], the estimated primary noise $\hat{d}(n)$ is delayed of one step to close the feedback loop and then stored in the vector $\mathbf{x}(n)$ (Eq. 2$)$. This vector, once filtered by means of the estimated secondary path $\hat{\mathbf{S}}(n)$ (Eq. 3 ), and the error signal $e(n)$ are used to update the adaptive FIR filter $\mathbf{W}(n)$ (Eq. 4). In the FIR updating equation, the step-size factor $\mu_{w}$ is used to setup the convergence and the velocity of the algorithm. The control signal $y(n)$ is then obtained by filtering $x(n)$ through the filter $\mathbf{W}(n)$ (Eq. 5). Finally, the forecast of the control signal at the error microphone $\hat{y}^{\prime}(n)$ is obtained by filtering the control signal $y(n)$ through the estimated secondary path $\hat{\mathbf{S}}(n)$ (Eq. 6).

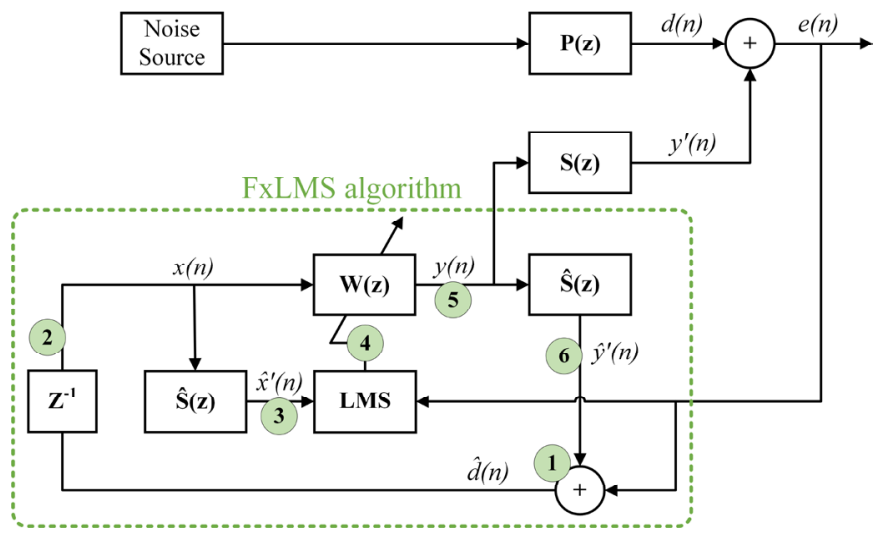

Fig. 2. Focus on the Fx-LMS Feedback algorithm implemented in the FPGA code.

$$
\begin{gathered}
\hat{d}(n)=y^{\prime}(n)+e(n) \\
\mathbf{x}(n)=\hat{\mathbf{d}}(n-1) \\
\hat{x}^{\prime}(n)=\hat{\mathbf{S}}(n)^{\mathrm{T}} \mathbf{x}(n) \\
\mathbf{W}(n+1)=\mathbf{W}(n)+\mu_{w} \mathbf{x}^{\prime}(n) e(n) \\
y(n)=\mathbf{W}(n)^{\mathrm{T}} \mathbf{x}(n) \\
\hat{y}^{\prime}(n)=\hat{\mathbf{S}}(n)^{\mathrm{T}} \mathbf{y}(n)
\end{gathered}
$$

In order to maximise the exploitation of the FPGA space, LUT memories were used to represent the $\mathbf{x}(n), \hat{\mathbf{x}}^{\prime}(n), \mathbf{y}(n)$ vectors and the $\hat{\mathbf{S}}(n)$ and $\mathbf{W}(n)$ filters. It should be noted that this approach is required to improve the length of the adaptive filter, even if it may reduce the number of samples of the error signal per second due to the high number of operations required for reading/writing memories. Thus, when designing mid/high frequency ANC systems, the use of standard vectors instead of memories is suggested by the Authors.

The flat sequence which is the core of the ANC FPGA algorithm is presented in Fig. 3. In the first cell the control signal $y(n)$ is generated by means of $\mathbf{x}(n)$, equal to $\hat{\mathbf{d}}(n-1)$, and 
$\mathbf{W}(n)$ and sent to the Analog Output module. Contemporarily, a fictitious memory (marked with the superscript ${ }^{\circ}$ in the code) is used to shift down of one unit the rows of the $\mathbf{y}(n-1)$ vector; thus, the new $y(n)$ value enters the first row of the vector in the second cell of the sequence, generating the vector $\mathbf{y}(n)$. In the third cell, the values of $\hat{y}^{\prime}(n), \hat{x}^{\prime}(n)$ and $\hat{d}(n)$ are calculated (see Eq. $6,3,1)$; in particular, $\hat{d}(n)$ is computed by means of the error signal $e(n)$ coming from the Analog Input module. Again, two fictitious memories are used in the fourth cell to convert the vectors $\hat{\mathbf{d}}(n-1)$ and $\hat{\mathbf{x}}^{\prime}(n-1)$ in $\hat{\mathbf{d}}(n)$ and $\hat{\mathbf{x}}^{\prime}(n)$ respectively by means of the $\hat{d}(n)$ and $\hat{x}^{\prime}(n)$ values. In the fifth cell the adaptive filter $\mathbf{W}(n)$ is updated in $\mathbf{W}(n+1)$ (see Eq. 4) and allocated in the fourth fictitious memory. In the last cell the values of the fictitious memories are transcribed in the original memories to get the vectors $\mathbf{W}(n+1)$, $\hat{\mathbf{d}}(n), \mathbf{y}(n), \hat{\mathbf{x}}^{\prime}(n)$ available for the next loop.
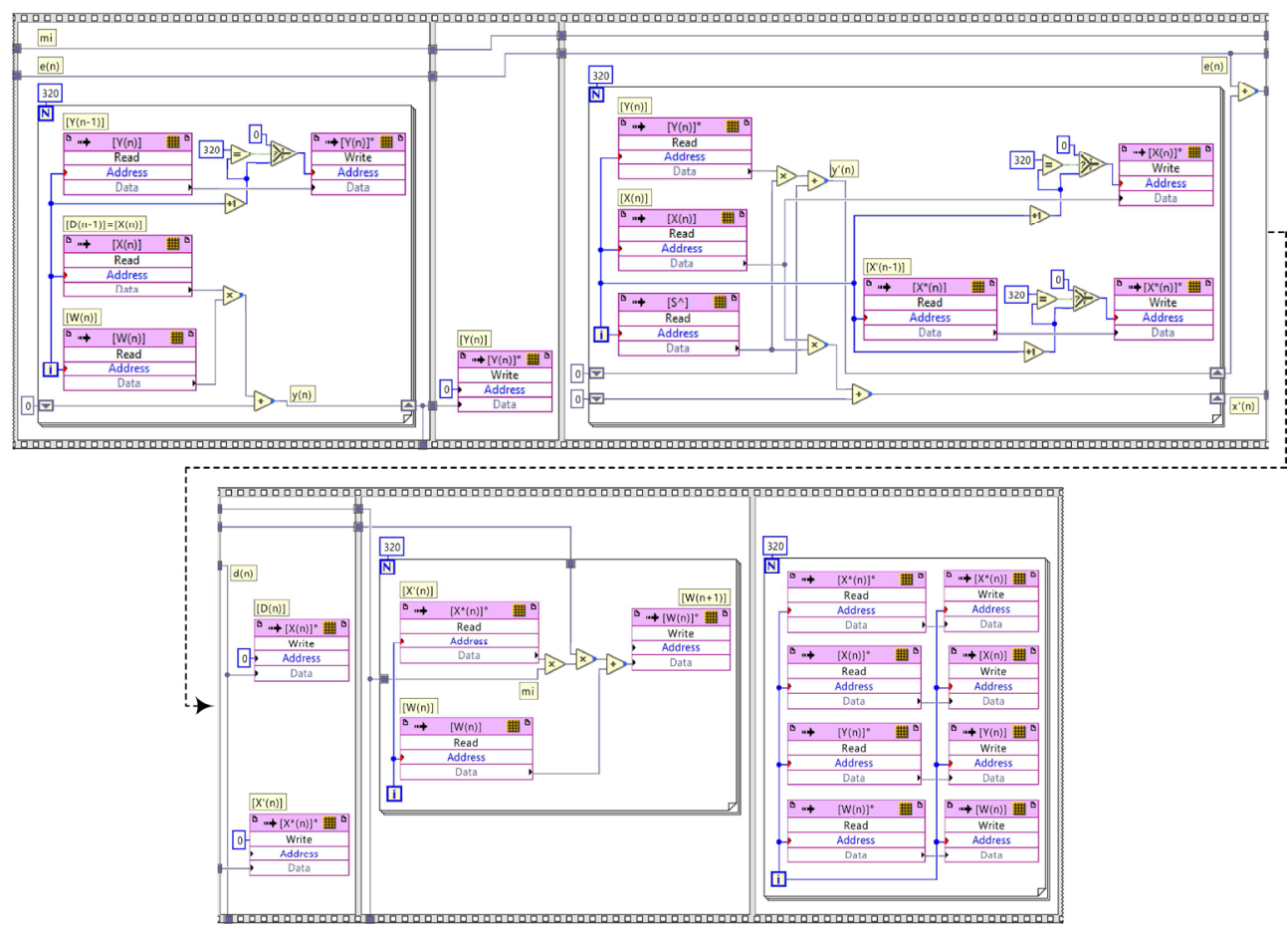

Fig. 3. Flat sequence for the generation of the control signal $y(n)$ and the update of the filter $\mathbf{W}(z)$.

The fixed-point 28-bit 6-integer data type was found to be a good trade-off between memory usage and algorithm effectiveness and was applied to all the operators in the code. A constant gain was used to reduce the amplitude of the error signal (volt) to best fit the selected data type. The length of the adaptive FIR filter $\mathbf{W}(n)$ was chosen by looking at the Literature experimental data. Typical values for Feedback algorithms are between 110 [28] and 512 [24] samples; moreover, best results were found by increasing filter length [20,29]. Thus, the Authors decided to fully exploit the available space on the FPGA, corresponding to 320 elements for both the $\mathbf{W}(z)$ and $\hat{\mathbf{S}}(z)$ filters. The frequency of the while loop hosting the flat sequence was set to $2 \mathrm{k}$ samples per second to match the target range of the noise spectrum. It is worth noting that the FIR filter in this configuration would be able to operate between $6.75 \mathrm{~Hz}$ (one sinewave, 320 samples) and about $250 \mathrm{~Hz}$ (forty sinewaves, eight samples each); sharper noises will not be filtered. The selected filter length is in accordance with subwoofers operating range, which states in average between 25 and $250 \mathrm{~Hz}$.

The effectiveness of the ANC algorithm depends on the accuracy of the estimation of the Secondary Path since it predicts the delay and the disturbance effects occurring between 
the control signal actuator and the error microphone. Both on-line and off-line methods can be followed to estimate the Secondary Path. In order not to compromise the length of the adaptive filter $\mathbf{W}(z)$, the Authors chose to off-line estimate the Secondary Path by means of a white noise reference signal, which is the most widespread approach in the Literature (Table 1). The scheme of the implemented algorithm is shown in Fig. 4, while Eq. 7-9 present the mathematical formulation. A dedicated LabVIEW FPGA code, based on the FxLMS algorithm shown in [3], was designed and deployed on the cRIO 9045 device (Fig. 5).

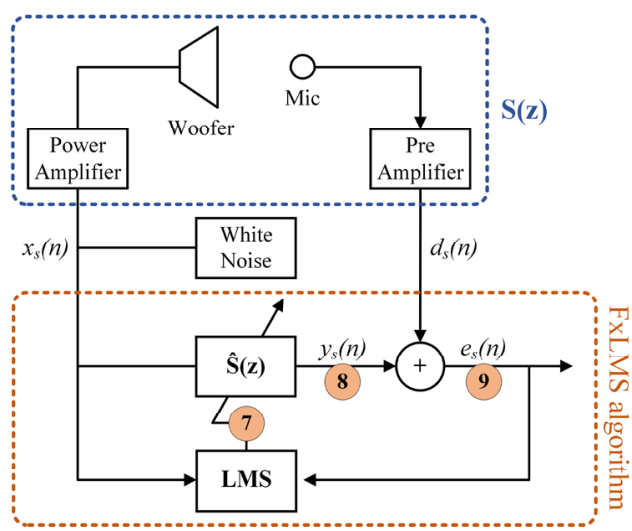

Fig. 4. Focus on the Fx-LMS Feedback algorithm for off-line Secondary Path estimation.
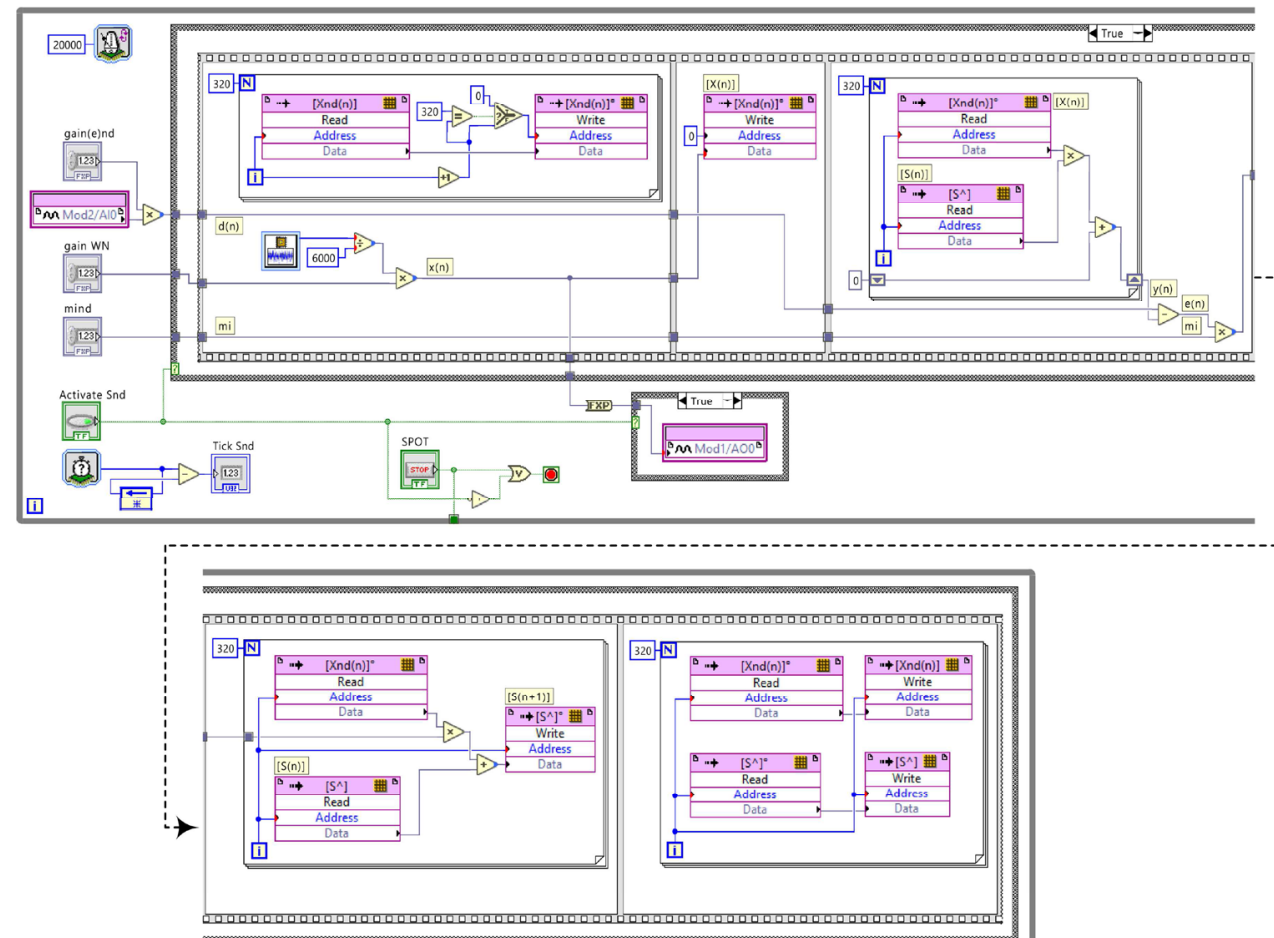

Fig. 5. While loop and flat sequence for the calibration of the Secondary Path static FIR filter $\hat{\mathbf{S}}(z)$. 


$$
\begin{gathered}
\hat{\mathbf{S}}(n+1)=\hat{\mathbf{S}}(n)+\mu_{s} \mathbf{x}_{\mathbf{s}}(n) e_{s}(n) \\
y_{s}(n)=\hat{\mathbf{S}}(n)^{\mathrm{T}} \mathbf{x}_{\mathbf{s}}(n) \\
e_{s}(n)=d_{s}(n)-y_{s}(n)
\end{gathered}
$$

It should be noted that the off-line Secondary Path estimation is well suited for quasisteady applications in which significant temperature (and thus, sound speed) variations are almost absent. In transient applications instead, the adoption of the on-line Secondary Path estimation is suggested [30]. The principles of on-line Secondary Path estimation are shown in [19] while instructions for an efficient implementation are presented in [20].

\subsection{Experimental validation of the ANC FPGA code}

Fig. 6 presents the experimental setup realized by the Authors at the LINEA Laboratory of the University of Florence for the validation of the developed LabVIEW FPGA code. The test rig was composed of a NI 9234 Analog Input module (1), communicating with a GRAS Type 46BD 1/4" microphone (2) (used as the error signal), and a NI 9263 Analog Output module (3) connected to a Pioneer GM-D9701 500W RMS single-channel amplifier (240 $\mathrm{Hz}$ high-pass crossover). A $350 \mathrm{~W}_{\mathrm{AES}}$ Ciare HSG200-22 subwoofer (4) was used to generate the control signal. A $40 \mathrm{~mm}$ piping system was used to connect the engine muffler to the control subwoofer by means of a Y-joint (5). A $60 \mathrm{~mm}$ length cylinder insert of rock wool was inserted in the $1 / 2$ " silicon pipe to protect the microphone from exhaust gas (6).

A $2.1 \mathrm{Lt} 50-\mathrm{kW}$ four-stroke four-cylinder heavy-duty diesel engine (7) was organized at the test bench and ran at idle condition $(800 \mathrm{rpm})$. T- and K-type thermocouples, connected to a NI 9211 module (8), were used to monitor temperature at the microphone, at the speaker outlet and inside the exhaust pipe to avoid parts damage.
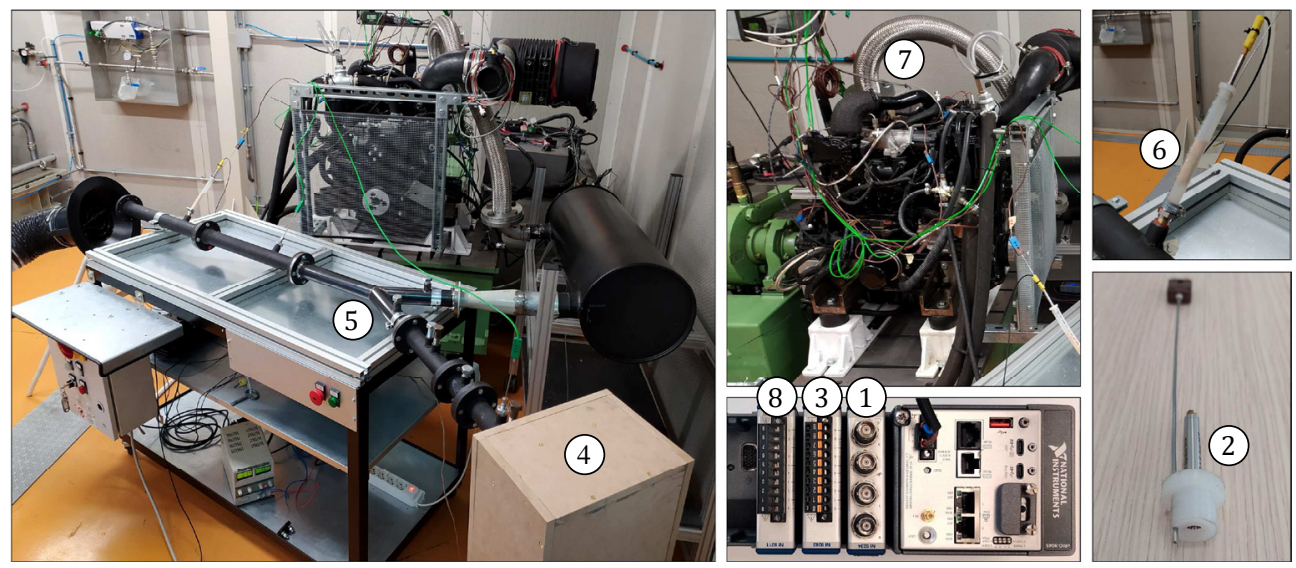

Fig. 6. Experimental setup developed by the Authors to test and calibrate the FPGA ANC algorithm.

Instabilities were found on the baseline Fx-LMS algorithm in steady-state operation. To improve algorithm stability without altering the filter updating equation (Eq. 10), the step size $\mu_{w}$ was divided by the difference between the error signal (in volt) achieved with the ANC system turned off, $e_{\max }$, and the actual error signal with ANC turned on, $e(n)$ (eq. 11).

$$
\begin{gathered}
\mathbf{W}(n+1)=\mathbf{W}(n)+\mu_{w}^{\prime}(n) \mathbf{x}^{\prime}(n) e(n) \\
\mu_{w}{ }^{\prime}(n)=\mu_{w} /\left|e_{\max }-e(n)\right|
\end{gathered}
$$


The developed ANC system was found to be able to abate (at the error microphone) up to more than $30 \mathrm{~dB}$ at the engine firing frequency $(26.7 \mathrm{~Hz})$ and about $5 \mathrm{~dB}$ at the second harmonic $(53.4 \mathrm{~Hz})$, for an average abatement of $17.8 \mathrm{~dB}$ in steady-state operation (20 min) between the same mechanical exhaust configuration with ANC turned off and on (Fig. 7).

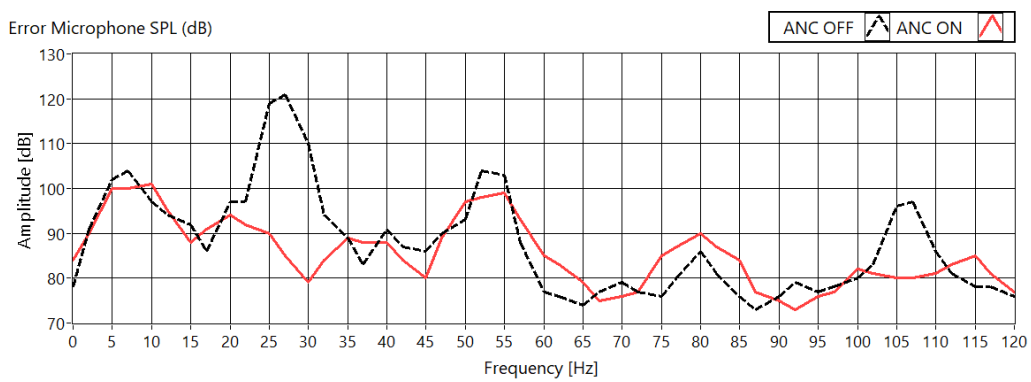

Fig. 7. SPL at the error microphone (LabVIEW screenshot) during calibration ( $800 \mathrm{rpm}$, no load).

\subsection{Deployment of the ANC system on a 50-kW industrial genset}

Once validated the FPGA code, the Authors decided to test the developed ANC system on a commercial industrial genset in a semi-anechoic chamber to evaluate the effectiveness on the surrounding ambient. The final ANC system prototype (Fig. 8) was thus specifically developed to be housed in the exhaust compartment of a 50-kW four-stroke diesel genset.
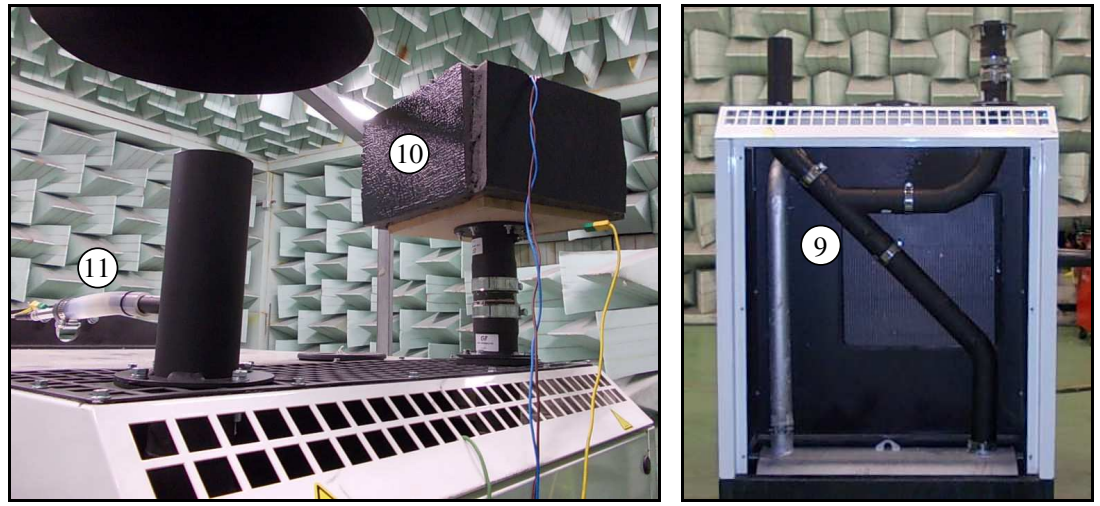

Fig. 8. Details on the layout of the ANC system, error microphone enclosure and subwoofer box.

The diameter of the ANC piping system was set to $80 \mathrm{~mm}$ to be coupled with the preexistent muffler outlet. A 45 degrees branch pipe (9) was used to fillet the control subwoofer to the main ANC pipeline. This piping configuration was essentially adopted for layout simplicity; anyway, it should be noted that preliminary experimental tests performed at the LINEA Laboratory on a narrowband noise between 50 and $300 \mathrm{~Hz}$ showed a $1.5 \mathrm{~dB}$ lower ANC effectiveness in average by using a T-joint configuration, while no sensitive difference was observed between the Y-joint and the branch pipe.

The subwoofer, enclosed in a bass-reflex wood case (110/45 mm back/front depth), was set above the compartment roof to distribute weight upon the structure and to remove it from the hot air of the exhaust compartment (10. The external walls of the subwoofer case were insulated to avoid sound losses. The flange of the subwoofer pipe was designed to accommodate a $3 \mathrm{~mm}$ thickness flat cartridge of melamine enclosed in a wire matrix. This solution was adopted to avoid thermal stress which may unglue the subwoofer cone. 
A BSWA 1/2" MPA215 microphone (135 dB saturation, $80^{\circ} \mathrm{C}$ max temperature) was used as the error microphone, after being enclosed in a $200 \mathrm{~mm}$ length silicon tube. In order to protect the microphone from thermal stress and to prevent pre-amplifier saturation (intube SPL at full load would exceed $140 \mathrm{~dB}$ ) a $60 \mathrm{~mm}$ cylinder insert of melamine was used (11). K-type thermocouples were installed on the branch joint, at the error microphone and at the subwoofer box (both front side and back side).

Ambient noise measurements were performed by means of a Delta Ohm HD 2110L phonometer on the twelve parallelepiped points described by the UNI EN ISO 11204:2010.

Investigations on the stability and effectiveness of the developed FPGA code highlighted that the absence of a dedicated cooling system for the subwoofer box may bring to a too high temperature inside the box in steady-state operation for high current absorption values, which may even lead to the break of the coils. In order to limit the current absorption, the filter update equation (Eq. 12) was further revised introducing a leakage factor $v$.

$$
\begin{gathered}
\mathbf{W}(n+1)=v \mathbf{W}(n)+\mu_{w} \mathbf{x}^{\prime}(n) e(n) \\
v=1-\mu_{w} \gamma
\end{gathered}
$$

The parameter $\gamma$ which defines the leakage factor (Eq. 13) was properly tuned to achieve a maximum power absorption of about $120 \mathrm{~W}$ in steady-state conditions, which was found to be a suited value in order not to stress the subwoofer coils and cone. Further information on implementation and benefits of the Leaky Fx-LMS algorithm can be found in [31-33].

\section{Results}

Preliminary results achieved in the semi-anechoic chamber showed a good potential for reducing combustion noise $(50 \mathrm{~Hz})$ and the second harmonic $(100 \mathrm{~Hz})$ at engine full-load condition by means of the developed ANC prototype. In fact, the overall SPL (linear $\mathrm{dB}$ ) at the error microphone was attenuated of $14.6 \mathrm{~dB}$ by switching on the ANC algorithm (for the same exhaust branch pipe layout) while absorbing about $250 \mathrm{~W}$ at the subwoofer coils.

However, the high current absorption and the absence of a dedicated cooling system for the subwoofer box have brought the temperature of the air surrounding the subwoofer cone near $60{ }^{\circ} \mathrm{C}$, causing the ungluing of the subwoofer cone. To avoid this issue, the maximum power absorption was limited to about $120 \mathrm{~W}$ through the adoption of a leakage factor in the ANC algorithm (see Eq. 12 and 13). Fig. 9 presents the real-time fast Fourier transform of the microphone error signal after about ten minutes of steady-state operation, in which an overall attenuation of $11.8 \mathrm{~dB}$ over the entire sound spectrum was achieved.

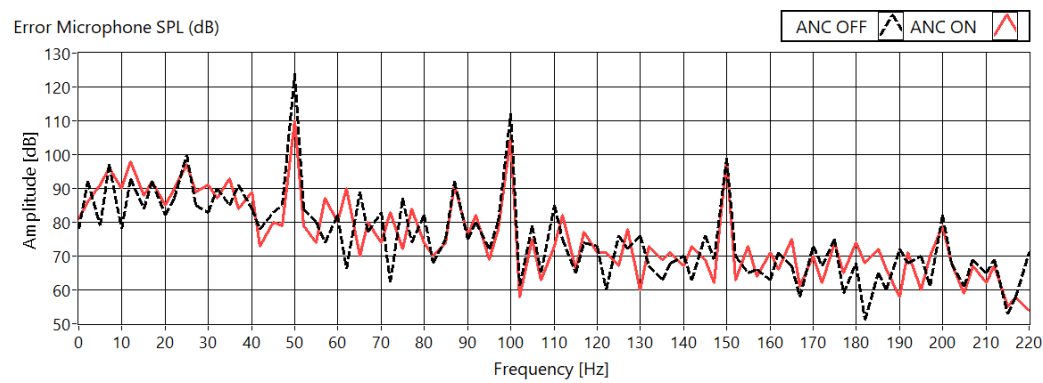

Fig. 9. Error microphone measurement (subwoofer limited to $120 \mathrm{~W}$, engine at $1500 \mathrm{rpm}$, full load).

In order to understand the effectiveness of the developed ANC system, the standard exhaust configuration (vertical pipe) must be preliminarily compared to the branch pipe 
layout, without turning on the ANC algorithm. Fig. 10 presents the SPL (dB) measurements obtained at a distance of one meter from the exhaust compartment and 1.5 meters from the basement (measurement point no. 4), which can be considered as the zone most affected by low-frequency noises.

As it can be seen, the sole mechanical piping layout (black diagonal brick bars) reduced the SPL in the $50 \mathrm{~Hz}$ third octave band of $2.3 \mathrm{~dB}$ (linear); however, it also introduced resonance effects at $100 \mathrm{~Hz}$, which increased the SPL of $8 \mathrm{~dB}$ in this specific band. This feature was not crucial for the investigated engine since the combustion noise is $19.3 \mathrm{~dB}$ higher than the $100 \mathrm{~Hz}$ harmonic at the measurement point no. 4. In fact, an overall $5.4 \mathrm{~dB}$ abatement, averaged on the whole noise spectrum, have been achieved at that measurement point by turning on the ANC algorithm, as a consequence of the $6.8 \mathrm{~dB}$ reduction in the 50 $\mathrm{Hz}$ band (red weave bars). However, generally speaking, this aspect is crucial to be taken into account since the ANC algorithm was not able to bring back the $100 \mathrm{~Hz}$ noise to the original engine level, showing a $5.7 \mathrm{~dB}$ higher noise in the $100 \mathrm{~Hz}$ band than the baseline configuration.

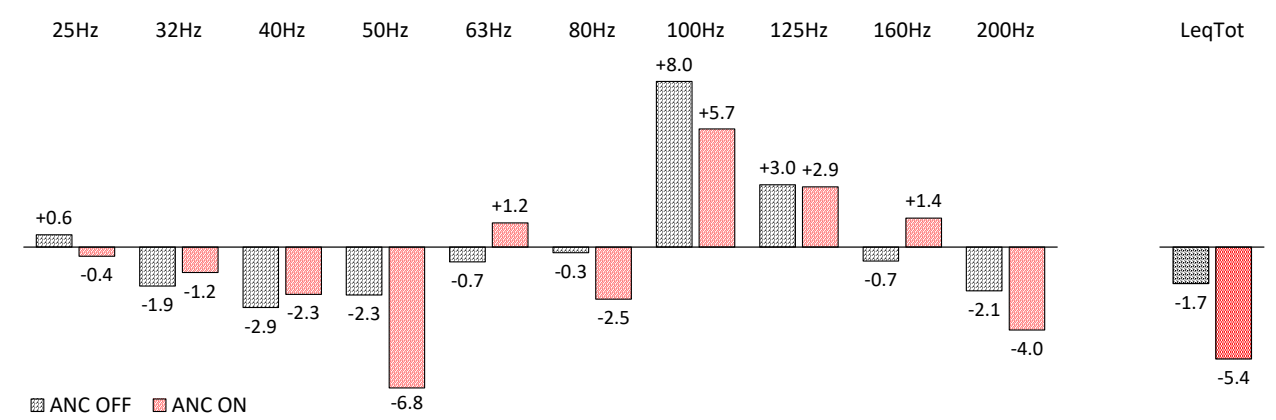

Fig. 10. SPL (dB) at distance of 1 meter from exhaust compartment (difference from baseline genset).

Considering instead the SPL (linear $\mathrm{dB}$ ) averaged on the 12 measurement points of the parallelepiped (UNI EN ISO 11204:2010), the ANC noise reduction effectiveness was found to be sensibly lower, achieving a $2.6 \mathrm{~dB}$ overall noise reduction (Fig. 11, red weave bars). This behaviour can be explained considering that low-frequency noise is predominant in the exhaust compartment vicinity, while moving toward the intake compartment midrange frequency noises, due to ventilation and air flushing, become more relevant.

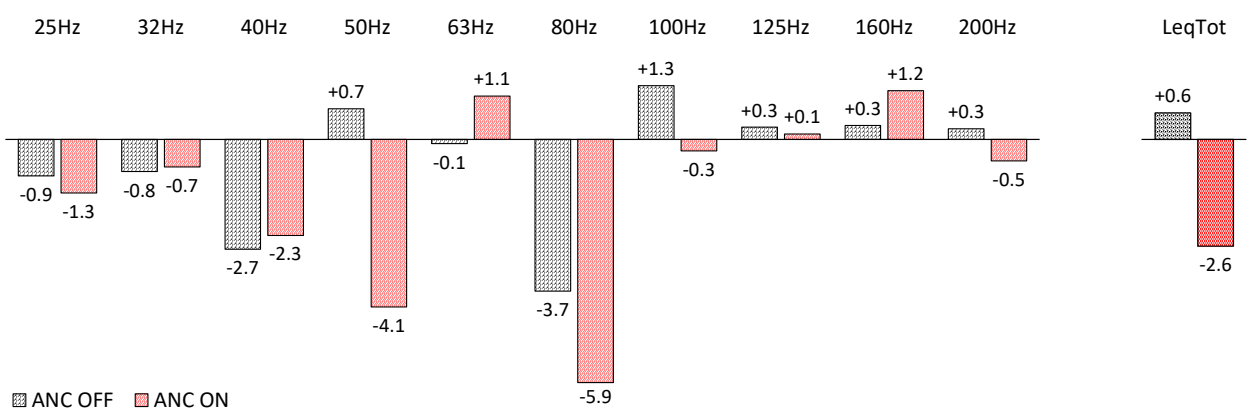

Fig. 11. SPL (dB) averaged on the 12 twelve parallelepiped points (difference from baseline genset).

It is worth noting that the aforementioned resonance effect in the $100 \mathrm{~Hz}$ third octave band due to the introduction of the branch pipe loses consistency when averaging the SPL on the 12 measurement points and tends to disappear by turning on the ANC algorithm. This behaviour can be explained considering that the vibrations which are introduced in the 
exhaust system by adding the branch pipe become imperceptible by moving away from the exhaust compartment.

Finally, results clearly show that the Feedback algorithm was able to adequately operate on the first and second harmonics of the genset noise spectrum; however, it was not found able to take action on the other noise bands. Indeed, the ANC system led to a slightly higher SPL for what concern the $40 \mathrm{~Hz}, 63 \mathrm{~Hz}$, and $160 \mathrm{~Hz}$ third octave bands (Fig. 10 and Fig. 11). In order to understand this behaviour, the noise spectrum of the baseline genset should be compared to the operating principle of the ANC algorithm. Figure 12 presents the range of interest of the baseline third octave spectrum at the measurement point no. 4 and that averaged on the twelve points of the parallelepiped (bands are evaluated as the percentage of the SPL of the noisiest band of the point no.4 spectrum). As it can be seen, the $50 \mathrm{~Hz}$ band prevails on the whole spectrum, especially considering that the SPL is measured in $\mathrm{dB}$, thus in a logarithmic scale; even the $100 \mathrm{~Hz}$ band loses consistency at the measurement point no. 4.

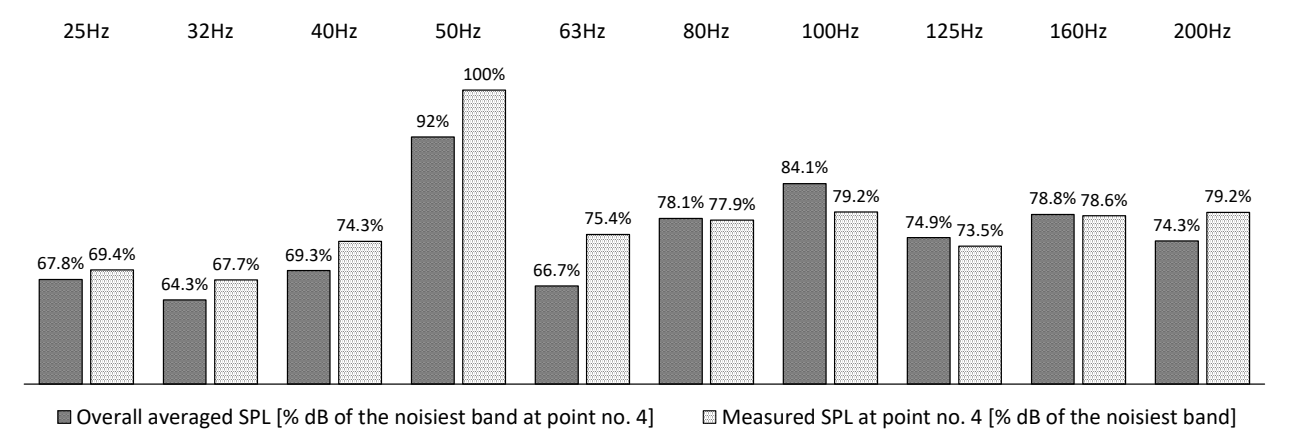

Fig. 12. Baseline genset noise spectrum (\% of the noisiest band of the point no. 4 spectrum).

As presented in Section 2.1, the ANC algorithm operates by means of the microphone voltage continuous signal, which is used as the error signal. Thus, it is not able to directly act on each frequency band; on the contrary, it operates only on those frequencies which lead to the reduction of the overall error signal in volt (and not $\mathrm{dB}$ ). This characteristic explains why the developed ANC system is capable of operating only on the $50 \mathrm{~Hz}$ and 100 $\mathrm{Hz}$ bands (as it can be clearly seen in Fig. 9). It should however be noted that, after all, the other frequency bands do not significantly influence the overall SPL of the genset; thus, the adoption of broadband ANC algorithms, based on the decomposition of the noise source in different frequency bands [34], does not seem to be necessary for this sort of application.

\section{Discussion}

The ANC prototype developed by the Authors has performed properly since the results are aligned with the Literature (Table 1), especially considering the imposed limit on the subwoofer power (current) absorption. However, several matters, that should be evaluated when designing any ANC prototype, must be accounted to improve the reliability and the effectiveness of the system.

The first consideration relates to the resonance effect of the branch pipe. The Helmholtz effect was found to be favourable around $50 \mathrm{~Hz}$; however, the adoption of a branch pipe led to a considerable higher noise in the $100 \mathrm{~Hz}$ band in the exhaust compartment vicinity. This issue was not critical in this study since the two third octave bands differ for more than 19 $\mathrm{dB}$ in the baseline genset configuration; however, attention should be paid when the main noise frequency is comparable in terms of amplitude to its harmonics. 
The second criticality concerns the electromechanical stability. The prototype, by means of the leakage factor, worked for 40 minutes so as to perform parallelepiped measurements without encountering algorithm instability. However, the noise attenuation at the error microphone decreased from 11.8 to $11.4 \mathrm{~dB}$ during the operating period, while the current absorption increased from 6.2 to 9.6 amps. This feature can be imputed to the overlap of the increased subwoofer impedance, due to the higher temperature of the coils, and the loss of performance of the subwoofer melamine cartridge.

Focusing on the behaviour of the cartridge, by injecting a $50 \mathrm{~Hz}$ pure tone (for the same current absorption of 6.1 amps) from the control speaker with the engine turned off, a $2 \mathrm{~dB}$ lower signal was measured at the error microphone by introducing the $3 \mathrm{~mm}$ thickness clean melamine shield (compared to the free pipe), despite the low-pass attitude of the material. This feature leads to a higher current absorption, increasing box temperature. Moreover, replacing the clean cartridge with a dirty one (about two hours of discontinuous use), a $2.7 \mathrm{~dB}$ lower signal was measured at the error microphone for the same generated tone. Thus, the use of porous phono-absorbing materials (e.g., melamine or rock wool) may lead to ANC system ineffectiveness and subwoofer overheating in long-time steady-state operations. Thus, the use of other materials, e.g., silicon sleeves, should be investigated to avoid fouling effects and to improve low-pass properties of the insulating cartridge.

Another note concerns the melamine insert for protecting the microphone, since the insulating material influences the quality of the error signal. The insert is required to avoid microphone thermal stress and to prevent pre-amplifier saturation, but it also modifies the shape of the error signal. Phono-absorbing porous materials are a good choice when abating low-frequency noises since they act as low-pass filters, thus excluding uninteresting frequencies. However, too thick inserts could influence even low-tone error signals, while too thin ones may not be enough to prevent from microphone saturation; thus, the insulating material and insert thickness/density should be carefully chosen.

The last critical feature concerns the control signal, which must be limited not to overheat the subwoofer coils. Since a saturator on the output peak-to-peak voltage cannot be used (it would lead to a square wave signal, thus increasing the RMS voltage) the use of a leakage factor was required. It is worth noting that a proper calibration process must be performed since it may strongly reduce ANC effectiveness. Moreover, the adoption of an air-cooled bass-reflex case is suggested to ensure air renewal inside the subwoofer box.

Finally, it must be noted that Feedback ANC systems cannot be considered as the sole technology for improving the noise abatement potential in industrial generators. In fact, genset sound spectrum is also composed of mid-frequency noise related to ventilation and air flushing, which may become predominant while activating the in-tube ANC system. Thus, passive solutions and/or open-space 3-D ANC systems should be adopted to reduce further the genset overall SPL.

\section{Conclusions}

The present study aimed at investigating the potential of the Feedback ANC technology for attenuating the engine noise at the exhaust of mid-size industrial gensets. A dedicated LabVIEW FPGA code was developed by the Authors and implemented in a 7K70T FPGA to acquire the residual noise and manage the control subwoofer. The Fx-LMS algorithm was used to update the ANC FIR filter. The ANC FPGA code was calibrated at the engine test facility of the University of Florence by using a four-cylinder $2.1 \mathrm{Lt}$ heavy-duty diesel engine at $800 \mathrm{rpm}$ and no load; then, it was installed inside the exhaust compartment of a $50-\mathrm{kW}$ industrial genset and tested in a semi-anechoic chamber at $1500 \mathrm{rpm}$ and full engine load for more than 40 minutes. Melamine shields have been applied to the microphone and the subwoofer cone to prevent thermal damage and fouling. 
The ANC prototype performed satisfactorily, achieving an overall attenuation of 11.8 linear $\mathrm{dB}$ over the entire sound spectrum at the error microphone in steady-state operation by activating the ANC algorithm (for the same branch pipe exhaust layout). Comparing to the baseline genset exhaust, an overall $5.4 \mathrm{~dB}$ abatement was measured at one meter from the exhaust compartment and 1.5 meters from the basement; furthermore, a $2.6 \mathrm{~dB}$ overall noise reduction was observed by performing a 12-points parallelepiped test.

Several challenges, however, have been addressed during tests. The ANC system in fact has shown a higher effectiveness by increasing the subwoofer current absorption; however, cone ungluing issues required the power absorption to be limited. This issue was solved by adopting a leaky ANC algorithm. Furthermore, resonance effects due to the layout of the branch pipe were observed in the $100 \mathrm{~Hz}$ band. Finally, melamine cartridges were found to be sensitive to fouling, reducing low-pass properties. These issues highlight the importance of the mechanical design in ANC systems for engine exhaust application; in particular, the use of dedicated cooling systems for the subwoofer box are suggested.

Further investigations on the material of the insulating cartridges and the design of bassreflex boxes are suggested for future studies, together with a comparison between feedback and feedforward algorithms to evaluate the best ANC algorithm for gensets.

\section{Acronyms and abbreviations}

$\begin{array}{ll}\gamma & \text { Leaky Fx-LMS calibration parameter } \\ \mu_{s} & \text { Step size of the } \hat{\mathbf{S}}(z) \text { filter update equation } \\ \mu_{w} & \text { Step size of the W(z) filter update equation } \\ v & \text { Leakage Factor } \\ d(n) & \text { Noise source estimation } \\ d_{s}(n) & \text { Microphone signal (Secondary Path estimation algorithm) } \\ e(n) & \text { Error microphone signal } \\ e_{\max } & \text { Maximum error signal with ANC turned off } \\ e_{s}(n) & \text { Error signal (Secondary Path estimation algorithm) } \\ x(n) & \text { Delayed estimated noise source } \\ x_{s}(n) & \text { White noise signal (Secondary Path estimation algorithm) } \\ y(n) & \text { Control signal } \\ y_{s}(n) & \text { Filtered white noise signal (Secondary Path estimation algorithm) } \\ \mathbf{S}(z) & \text { Secondary Path } \\ \hat{\mathbf{S}}(z) & \text { Estimated Secondary Path FIR filter } \\ \text { W }(z) & \text { ANC adaptive FIR filter } \\ \text { ANC } & \text { Active Noise Control } \\ \text { FX-LMS } & \text { Filtered-X Least Mean Square algorithm } \\ \text { FIR } & \text { Finite Impulse Response filter } \\ \text { FPGA } & \text { Field Programmable Gate Array } \\ \text { LUT } & \text { Look-Up Table } \\ \text { NI } & \text { National Instruments } \\ \text { SPL } & \text { Sound Pressure Level } \\ & \end{array}$

\section{References}

1. M. L. Munjal, Int. J. Acoust. Vib. 18, (2013)

2. E. Arnold, W. Frazer, and W. J. J. Hoge, Development of a Prototype Active Muffler for the Detroit Diesel 6V-92 TA Industrial Engine (SAE International, Warrendale, PA, 1991) 
3. S. M. Kuo and D. Morgan, Active Noise Control Systems: Algorithms and DSP Implementations, 1st ed. (John Wiley \& Sons, Inc., USA, 1995)

4. M. Cuesta and P. Cobo, Appl. Acoust. 62, 513 (2001)

5. M. Anthony, C.-Y. Chang, and S. M. Kuo, in 2017 Asia-Pac. Signal Inf. Process. Assoc. Annu. Summit Conf. APSIPA ASC (2017), pp. 140-144

6. K. I. M. Heung-Seob, H. Jin-Seok, and O. H. Jae-Eung, JSME Int. J. Ser. C 41, 178 (1998)

7. F. Cotana and F. Rossi, J. Acoust. Soc. Am. 112, 2427 (2002)

8. S.-K. Lee, S. Lee, J. Back, and T. Shin, Appl. Sci. 8, 1394 (2018)

9. D. Miljkovi'c, Automatika 57, 1056 (2016)

10. J. Cheer and S. J. Elliott, Appl. Acoust. 105, 209 (2016)

11. Y. Kobayashi and H. Fujioka, Adv. Acoust. Vib. 2008, e253948 (2008)

12. J. Liang, T. Zhao, L. Zou, L. Zhang, and Z. Li, in 2015 Fifth Int. Conf. Instrum. Meas. Comput. Commun. Control IMCCC (2015), pp. 1394-1397

13. W. Oh, in 3rd Int. Conf. Innov. Eng. Technol. ICIET2016 August 5-6 2016 Bangk. Thail. (International Institute of Engineers, 2016)

14. Kuo-Kai Shyu and Cheng-Yuan Chang, IEEE Trans. Ind. Electron. 47, 444 (2000)

15. D. Bismor, K. Czyz, and Z. Ogonowski, Int. J. Acoust. Vib. 21, (2016)

16. S. M. Kuo and D. R. Morgan, Proc. IEEE 87, 943 (1999)

17. Y. Jiang, S. Chen, F. Gu, H. Meng, and Y. Cao, Appl. Acoust. 175, 107816 (2021)

18. Y. Kajikawa, W.-S. Gan, and S. M. Kuo, APSIPA Trans. Signal Inf. Process. 1, (2012)

19. L. J. Eriksson and M. C. Allie, J. Acoust. Soc. Am. 85, 797 (1989)

20. H. Hassanpour and P. Davari, Digit. Signal Process. 19, 241 (2009)

21. J.-D. Wu and M. R. Bai, Jpn. J. Appl. Phys. 39, 4982 (2000)

22. J. Shaw, WSEAS Trans. Math. 1, (2002)

23. F. Rossi, in Proc. Energy Environ. (Italy, 2002)

24. L. Wu, X. Qiu, and Y. Guo, Appl. Acoust. 81, 40 (2014)

25. I. D. Landau, R. Meléndez, L. Dugard, and G. Buche, IEEE Trans. Control Syst. Technol. 27, 872 (2019)

26. I. Isranuri, Alfisyahrin, and A. R. Nasution, IOP Conf. Ser. Mater. Sci. Eng. 308, 012011 (2018)

27. Y. Kajikawa, in 2011 8th Int. Conf. Inf. Commun. Signal Process. (2011), pp. 1-5

28. W. S. Gan, S. Mitra, and S. M. Kuo, IEEE Trans. Consum. Electron. 51, 975 (2005)

29. P. Kasbekar, A. Wisler, and I. Panahi, in 2013 Proc. IEEE Southeastcon (2013), pp. 1-5

30. P. Davari and H. Hassanpour, in 2008 IEEE Int. Conf. Ind. Technol. (2008), pp. 1-6

31. O. J. Tobias and R. Seara, in 2002 11th Eur. Signal Process. Conf. (2002), pp. 1-4

32. D. Bismor and M. Pawelczyk, Arch. Acoust. 41, 731 (2016)

33. L. Wu, X. Qiu, and Y. Guo, Mech. Syst. Signal Process. 106, 13 (2018)

34. L. Luo, J. Sun, and B. Huang, Appl. Acoust. 116, 229 (2017) 